\title{
Safety Evaluation for Pure Titanium by Lock-in Infrared Thermography
}

\author{
T. Matsunaga ${ }^{a,}{ }^{*}$, N. Nagashima ${ }^{a}$, T. Nojima ${ }^{a}$, and S. Sugimoto ${ }^{b}$ \\ ${ }^{\mathrm{a}}$ National Institute for Materials Science, Ibaraki, Japan \\ $\mathrm{b}_{\text {JFE Techno-Research Corporation, Kanagawa, Japan }}$
}

\begin{abstract}
The lock-in infrared thermography has been applied to an estimation of fatigue limits at several stress ratios, i.e., $R=-1,0.05$, and 0.5 , for commercially pure titanium (CP-Ti JIS grade 2) at room temperature. In the present study, frequencies were $1 \mathrm{~Hz}$ and $4 \mathrm{~Hz}$ at $R=-1$, and $7 \mathrm{~Hz}$ at $R=0.05$ and 0.5 . Tests at $R=-1$ showed a negligible frequency dependency of a fatigue limit of $250 \mathrm{MPa}$. Other tests at $R=0.05$ and 0.5 showed respective fatigue limits of $123 \mathrm{MPa}$ and $48 \mathrm{MPa}$. Tensile tests were performed to obtain a tensile strength and a proof stress. A fatigue limit diagram of the sample was plotted using the above values, showing a violation of the mod. Goodman line and the Soderberg line. Next, the diagram was revised using a stress starting plastic deformation shown by creep tests. It revealed a safety area of the new diagram is $35 \%$ smaller than that of the conventional safety standards.
\end{abstract}

\section{Introduction}

Development periods of new aircrafts become more than 10 years. Because an evaluation of a safety standard of a new material needs more than a year by standard mechanical tests, it occupies the large part of the development period. Recently, a new method, i.e., a lock-in infrared thermography [1-4], is considered for an estimation of a fatigue limit at a stress ratio $(R)$. For the method, a fatigue limit can be estimated in one day. Due to the reduction of safety evaluation time, it becomes the fatal method for aerospace industry and other fields. In addition, the lock-in infrared thermography is expected to estimate a fatigue limit. An infrared camera detects an irreversible temperature change including a thermo-elastic effect and an energy dissipation ( $\left.T_{\mathrm{E}}[\mathrm{K}]\right)$ during cyclic loading with a frequency $(f)$. The effects can separate by Fourier analysis because the frequency of energy dissipation is double, i.e., $2 f$, comparing with the cyclic loading and the temperature change by the thermo-elastic effect [4].

Then, the dissipated energy $\left(q\left[\mathrm{~J} / \mathrm{m}^{3}\right]\right)$ expresses using a following equation with $c$ is a specific heat and $r$ is a density of a material.

$$
q=c r T_{\mathrm{E}} \quad(1)
$$

It has been reported that the energy increases with increasing stress amplitude (see Fig. 1). In addition, increase rates of the dissipated energy are divided into two regions. The transition point has been corresponded to fatigue limit of metals and alloys. In the present study, the lock-in infrared thermography was applied for commercially pure titanium (CP-Ti, JIS grade 2). The fatigue limit estimated by the thermography compared to that obtained by standard fatigue tests to confirm the utility of the new estimation method of fatigue limit for CP-Ti at room temperature.

\section{Experimental procedures}

A grade of CP-Ti in the present study was JIS grade 2 which chemical composition is shown in Table 1. Grain size was about 20 mm, which is evaluated using the intercept length. Sample of the lock-in infrared thermography was a plate-type specimen with a thickness of $3 \mathrm{~mm}$ and a width of $3 \mathrm{~mm}$. One surface was black-sprayed to matt metallic luster because an accuracy of temperature measurement increased. Specimen surface was polished an emery paper with $\# 600$ with polished direction corresponded to the loading direction.

Table 1. Chemical composition of the tested CP-Ti JIS grade 2.

\begin{tabular}{|c|c|c|c|c|c|c|}
\hline & $\mathrm{H}$ & $\mathrm{O}$ & $\mathrm{N}$ & $\mathrm{Fe}$ & $\mathrm{C}$ & $\overline{\mathrm{Ti}}$ \\
\hline mass $\%$ & 0.005 & 0.11 & $<0.01$ & 0.10 & 0.01 & Bal. \\
\hline
\end{tabular}

The infrared camera was one of the FLIR-SC7000 series. Frame rate of the camera was $99 \mathrm{~Hz}$. Fatigue tests were performed by staged increases of stress amplitude with $1 \mathrm{~Hz}$ and $4 \mathrm{~Hz}$ at $R=-1$, and $7 \mathrm{~Hz}$ at $R=0.05$ and 0.5 at room temperature. Waveform was sinusoidal. The ranges of stress amplitude were $100-340 \mathrm{MPa}$ for the tests at $R=-1,20-190 \mathrm{MPa}$ at $R=0.05$ and $10-100 \mathrm{MPa}$ at $R=0.5$. Shooting time of the infrared camera was $30.3 \mathrm{~s}$ to obtain 3000 pictures during one shoot. In addition, the thermography measurements were performed three times at one stress amplitude.

The dissipated energy was evaluated using the obtained pictures by a software (CERIP Inc. Altair LI). The software revealed the irreversible temperature change by the dissipated energy, $\mathrm{D} T_{\mathrm{E}}$. The energy was calculated using eq. (1) with $c(=0.523[\mathrm{~J} / \mathrm{g} \cdot \mathrm{K}])$ and $r\left(=4.51 \times 10^{6}\left[\mathrm{~g} / \mathrm{m}^{3}\right]\right)$ whose material constants were reported in ref. [5]. Next, the dissipated energy was plotted with stress amplitude as shown in Fig. 1. Although dissipated energy increased with increasing stress amplitude, there were two regions with different increasing rates.

Although specimen was round-bar type with $\mathrm{f}=3 \mathrm{~mm}$, standard fatigue tests were performed at $R=0.05$ and 0 at room temperature to compare the fatigue limit observed by the infrared camera. Frequency range in the tests was $1 \mathrm{~Hz}-30 \mathrm{~Hz}$

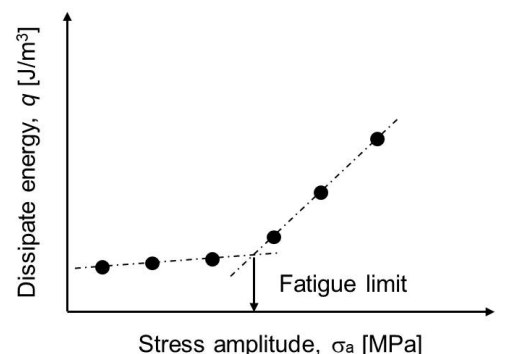

Fig. 1 Schematic plot of relation with dissipated energy and stress amplitude. Fatigue limit is estimated as the transition point at the stress amplitude.

\section{Results and Discussion}

Figure 2 showed the dissipated energy functioned by stress amplitude for each test. For the tests at $R=-1$, the dissipated energy at $f=1 \mathrm{~Hz}$ was smaller than that at $f=4 \mathrm{~Hz}$ (see Fig. 2 (a)). It might result from the thermal diffusion to air jig, and so on occurs during the cyclic loading. However, the fatigue limits observed at both frequencies were almost same, i.e., ca. $250 \mathrm{MPa}$. For $\mathrm{CP}$-Ti, the low frequency such as $1 \mathrm{~Hz}$ was enough to observe the fatigue limit and the frequency dependency was negligible at the frequencies. Then, Fig. $2(\mathrm{~b})$ and $2(\mathrm{c})$ showed the results at $R=0.05$ and 0.5 , respectively. Although the dissipated energy decreased with increasing stress ratio, these conditions included the transition point of increase rate of dissipated energy. The estimated fatigue limits were $123 \mathrm{MPa}$ and $48 \mathrm{MPa}$ for $R=0.05$ and 0.5 , respectively. 

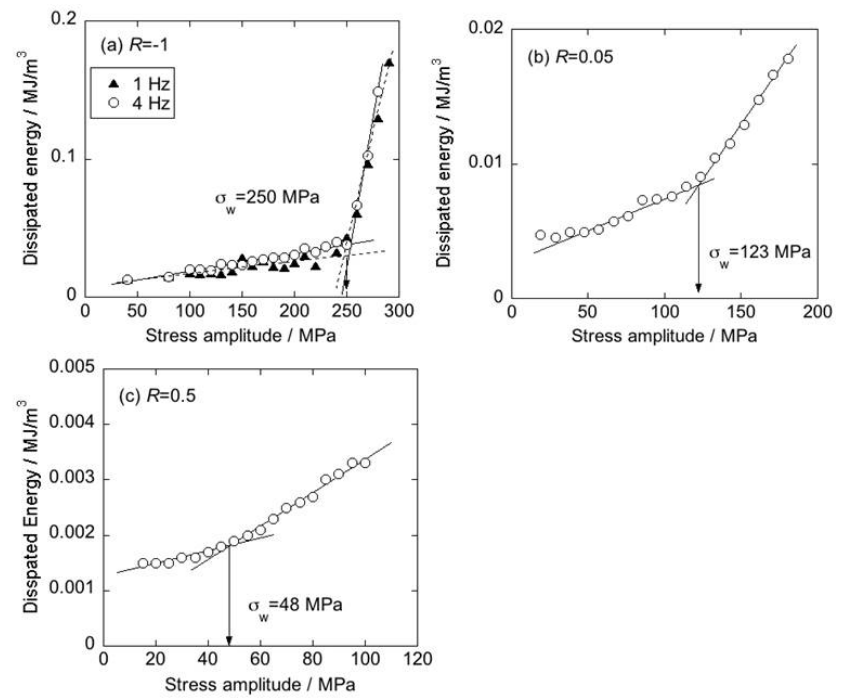

Fig. 2 Relation between dissipated energy and stress amplitude at $R=(\mathrm{a})-1$, (b) 0.05 , and (c) 0.5 for CP-Ti JIS grade 2 at room temperature.

Standard fatigue tests were performed at $R=0.05$ (closed circles) and 0 (open circles) to confirm the fatigue limit estimated using the infrared thermography. A frequency range of the fatigue tests was 1-30 Hz. S-N curve at the conditions was plotted in Fig. 3. The frequency dependency might be negligible because the plotted data did not show remarkable dispersion. In addition, the fatigue limit by the standard fatigue tests might be about $140 \mathrm{MPa}$ because the sample tested at the stress was not ruptured at $10^{7}$ cycles. The stress was almost same as the fatigue limit estimated using the infrared thermography. Although the number of the sample was only one, the lock-in infrared thermography can estimate the fatigue limit of CP-Ti and is available for the quick safety evaluation.

The fatigue limits estimated by the lock-in infrared thermography were plotted in a fatigue limit diagram of CP-Ti. The diagram contained a tensile strength and a proof stress evaluated by tensile tests. The mod. Goodman line and the Soderberg line were drown using the data. However, CP-Ti did not obey the conventional safety standards. Figure 4 also showed a stress stating plastic deformation which is obtained by creep tests. Using the value, new safety standard was drawn. It means the conventional safety standards, i.e., the mod. Goodman line and the Soderberg line, have been overestimated. Especially, the fatigue limit decreased at the $R=0.5$ remarkably. In the condition, mean stress is loaded during the tests. Because it has been reported that CP-Ti shows creep behavior at room temperature [6,7], accumulated strain during the fatigue tests might affect the fatigue limits. Therefore, the present study showed the new safety standard using the lock-in infrared thermography and revealed the safety level decreased comparing with the conventional safety standards. In addition, the new safety area became about $65 \%$ of that shown by the mod. Goodman line.

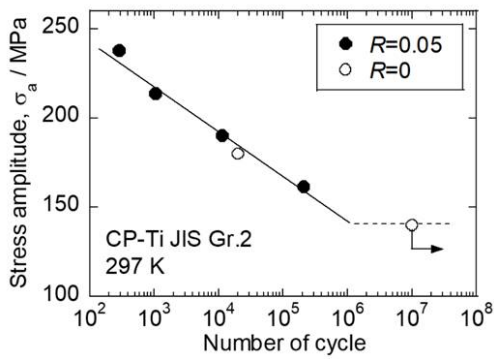

Fig. 3 S-N curve of CP-Ti JIS grade 2 at $R=0.05$ and 0 . The data is obtained by the standard fatigue tests at room temperature.

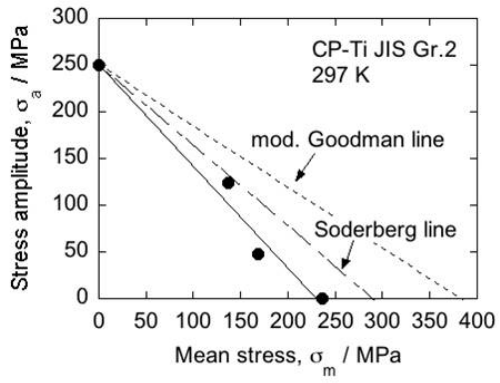

Fig. 4 Fatigue limit diagram of CP-Ti JIS grade 2 at room temperature. The mod. Goodman line and the Soderberg line are drawn in the figure.

Finally, it is discussed that the reduction resulted from the occurrence of creep at room temperature. Room temperature creep has been reported in hexagonal close-packed metals and alloys, i.e., Ti alloys, $\mathrm{Mg}$ alloys, $\mathrm{Zn}$ and so on [6-11] because of low symmetricity of the crystal structure. Therefore, these materials have low number of equivalent slip systems, i.e., only three in basal plane, which leads to dislocation arrays without tangles in grains. The unique dislocation structure results in low work-hardening and then strain accumulates under constant loading. Similar loading condition was archived for the fatigue tests at $R=0.05$ and 0.5 . In these cases, positive mean stresses were loaded during fatigue tests, which can regard as the constant loading. In fact, strain accumulation occurred in the standard fatigue test at $R=0.05$ and 0.5 in the present study. Therefore, the room temperature creep with the unique dislocation structure might affect fatigue limit in CP-Ti

\section{Conclusions}

In the present study, the lock-in infrared thermography was applied for the safety evaluation of CP-Ti JIS grade 2 at room temperature. The fatigue limits were estimated by the new method to modify the fatigue limit diagram of the material. The detailed conclusions are follows.

(1) The frequency dependency of the fatigue limit estimated by the infrared thermography was negligible in CP-Ti. The frequency range of the fatigue tests was 1-10 Hz in the present study.

(2) The fatigue limit estimated by the infrared thermography at $R=0.05$ corresponded to that evaluated by the standard fatigue tests. Because the testing time of the thermography was only one day for the estimation, the new method might decrease the time for safety evaluation drastically.

(3) According to the fatigue limits at $R=-1,0.05$ and 0.5 estimated by the lock-in infrared thermography, the fatigue limit diagram of CP-Ti was modified as shown in Fig. 4. Because the Mod. Goodman line and the Soderberg line overestimated the safety level, the new safety standard of CP-Ti was proposed in the present study. This line showed the decrease of safety area, which is only $65 \%$ comparing with the mod. Goodman line. 
The authors are grateful for fundamental supports from the Light Metal Educational Foundation Inc. and Innovative Science and Technology Initiative for Security, ATLA, Japan.

\section{References}

1. M.P. Luong, Mech. Mater. 27, 453 (1998).

2. G. La Rosa, A. Risitano, Int. J. Fatigue. 22, 65 (2000).

3. D. Shiozaki, T. Inagawa, T. Washio, T. Sakagami, Proc. Struc. Integrity. 2, 2019 (2016).

4. A. Akai, D. Shiozawa, S. Funazo, K. Chizaka, T. Sakagami, J. Soc. Mater. Sci. Japan. 67, 1036 (2018). Japanese.

5. H. Qin, J. J. Jonas, H. Yu, N. Brodusch, R. Gauvin, Acta Mater. 71, 293 (2014)

6. T. Matsunaga, T. Kameyama, K. Takahashi, E. Sato, Mater. Trans. 50, 2858 (2009).

7. T. Matsunaga, T. Kameyama, K. Takahashi, E. Sato, Mater. Trans. 50, 2865 (2009).

8. B.C. Odegard, A.W. Thompson, Metall. Trans. A. 5, 1207 (1974).

9. M.A. Imam, C.M. Gilmore, Metall. Trans. A. 10, 419 (1979).

9. T. Neeraj, D.H. Hou, G.S. Daehn, M.J. Mills, Acta Mater. 47, 1019 (2000).

10. T. Matsunaga, Y. Satoh, H. Abe, J. Nucl. Mater. 465, 358 (2015).

*Tetsuya Matsunaga: MATSUNAGA.Tetsuya@nims.go.jp 